\title{
Esteroides epidurales en el dolor radicular lumbar: particulados vs. no particulados. Esta es la cuestión
}

\author{
L. Cánovas Martínez
}

Jefa de la Unidad del Dolor. Complejo Hospitalario Universitario de Orense. España

Tagowski M, Lewandowski Z, Hodler J, Spiegel T, Goerres GW. Pain reduction after lumbar epidural injections using particulate versus non-particulate steroids: intensity of the baseline pain matters. Eur Radiol 2019. D0I: 10.1007/ s00330-019-06108-9.

La inyección epidural es una de las técnicas más utilizadas para el tratamiento del dolor lumbar irradiado a miembros inferiores debido a hernia discal, estenosis de canal o como consecuencia de una cirugía lumbar. La evidencia de la asociación de anestésicos locales y corticoides para el tratamiento de la radiculitis secundaria a hernia discal es buena y limitada para la utilización de anestésicos locales solos. La utilización de anestésicos locales y esteroides en el tratamiento de la radiculitis secundaria a estenosis de canal tiene una evidencia limitada (1).

Debido a la absorción más lenta de los esteroides particulados en comparación con los no particulados, los esteroides particulados se consideraron formulaciones más potentes y han sido elegidos preferentemente por los médicos especialistas en tratamiento intervencionista del dolor.

En este artículo de Tagowski y cols. (2), los autores compararon el alivio del dolor después de las inyecciones de esteroides epidurales lumbares guiadas por TAC, en pacientes con radiculopatía, utilizando esteroides particulados (triamcinolona) y esteroides no particulados (dexametasona), y analizaron los factores que afectaron a la efectividad de ambos tipos de esteroides. Este estudio observacional retrospectivo incluyó a 806 pacientes con radiculopatía lumbar y permitió establecer dos cohortes de 209 pacientes con alteraciones en RMN y TAC homogéneas par cada grupo.

Cuatro semanas después de la inyección epidural, la probabilidad alcanzar $\geq 50 \%$ de reducción del dolor fue la mitad en el grupo de dexametasona que en el grupo de triamcinolona $[\mathrm{OR}]=0,55 ; \mathrm{p}<0,012$ ]. La triamcinolona fue superior a la dexametasona en la radiculopatía lum- bar con dolor intenso $\geq$ de 7. Para el dolor leve a moderado, la dexametasona podría ser igualmente efectiva. La dexametasona reduce el dolor causado por hernia discal mucho mejor que el dolor causado por estenosis de canal. El efecto de triamcinolona no se vio influenciado por la intensidad del dolor ni las alteraciones anatómicas.

Tres comentarios al respecto; el primero de ellos es que el abordaje por TAC para la realización de una técnica epidural no se utiliza en la práctica clínica habitual, a lo que hay que añadir la alta radiación a la que se ven expuestos los pacientes del estudio. Sí se debe hacer con fluoroscopia.

En segundo lugar, los resultados podrían estar sesgados por la única utilización de la escala NRS (pain numerical rating scale] que cuantifica el dolor en leve, moderado o severo, pero que no tiene en cuenta el componente neuropático, posiblemente presente en muchos pacientes del estudio, que podría ser evaluado por una escala específica para dolor neuropático, así como el efecto de uno u otro esteroide en la mejoría de este componente [3].

Aunque las recomendaciones de consenso internacional y la FDA como observador incluyen una declaración en la que se dice que en una epidural transforaminal lumbar se debe usar un esteroide no particulado para la inyección inicial, también incorporan que un esteroide particulado podría considerarse en este contexto, por ejemplo, si el tratamiento inicial con un esteroide no particulado falla. Además, el uso de esteroides particulados en inyecciones interlaminares lumbares es aceptable, porque se minimiza el riesgo de inyección intraarterial con las normas de seguridad recomendadas en las guías (4). A pesar de esto, el esteroide particulado, triamcino- 
Iona, utilizado en este estudio, tiene una ficha técnica en España que contraindica su utilización por vía epidural e intradural [5]. Sin embargo, la práctica clínica, como la de este estudio, recoge que los esteroides particulados, y en concreto la triamcinolona, son más efectivos que los no particulados, como la dexametasona.

Al margen de los comentarios, los autores señalan que la intensidad del dolor basal condiciona el tipo de respuesta a un esteroide epidural, tanto particulado como no particulado. Necesitamos actualización de los consensos en la utilización de esteroides epidurales que definan mejor sus indicaciones y la elección de tipo de esteroide particulado vs. no particulado.

\section{BIBLIOGRAFÍA}

1. Benyamin R, Manchikanti L, Parr A, Diwan S, Singh V, Falco FJ, et al. The effestiveness of lumbar onterlaminar epidural injections in managing chronic low back and lower extremity pain. Pain Physician 2012;15(4):E363-E404.

2. Tagowski M, Lewandowski Z, Hodler J, Spiegel T, Goerres GW. Pain reduction after lumbar epidural injections using particulate versus non-particulate steroids: intensity of the baseline pain matters. Eur Radiol 2019. DOI: 10.1007/ s00330-019-06108-9 [Epub ahead of print].

3. Adebajo A, Fabule J. Management of radicular pain in rheumatic disease: insight for the physician. Ther Adv Musculoskel Dis 2012;4(3):137-47. DOI: 10.1177/1759720X12437180.

4. Rathmell JP, Benzon HT, Dreyfuss P, Huntoon M, Wallace M, Baker R, et al. Safeguards to prevent neurologic complications after epidural steroid injections: consensus opinions from a multidisciplinary working group and national organizations. Anesthesiology 2015;122(5):974-84. DOI: 10.1097/ALN.0000000000000614.

5. https://cima.aemps.es/cima/pdfs/es/ft/44901/ FT_44901.html.pdf 